\title{
Corela
}

Cognition, représentation, langage

12-1 | 2014

Vol. $12, \mathrm{n}^{\circ} 1$

\section{Evaluation critique des modèles graduels et non graduels de l'intégration syntaxique}

\section{Gilles Corminboeuf et Christophe Benzitoun}

\section{OpenEdition}

Journals

Édition électronique

URL : http://journals.openedition.org/corela/3480

DOI : $10.4000 /$ corela.3480

ISSN : 1638-573X

Éditeur

Cercle linguistique du Centre et de l'Ouest - CerLICO

Référence électronique

Gilles Corminboeuf et Christophe Benzitoun, «Evaluation critique des modèles graduels et non graduels de l'intégration syntaxique », Corela [En ligne], 12-1 | 2014, mis en ligne le 26 juin 2014 consulté le 01 mai 2019. URL : http://journals.openedition.org/corela/3480; DOI : 10.4000/ corela.3480

Ce document a été généré automatiquement le 1 mai 2019.

\section{(i)(2) (2)}

Corela - cognition, représentation, langage est mis à disposition selon les termes de la licence Creative Commons Attribution - Pas d'Utilisation Commerciale - Partage dans les Mêmes Conditions 4.0 International. 


\title{
Evaluation critique des modèles graduels et non graduels de l'intégration syntaxique
}

\author{
Gilles Corminboeuf et Christophe Benzitoun
}

\section{NOTE DE L'AUTEUR}

Une partie de cette étude est parue avec la référence suivante : Corminboeuf G. \& Benzitoun C. (2014), « Approches non gradualistes des liaisons de prédications : l'exemple des modèles macro-syntaxiques », Langue française 182, 43-58.

\section{Introduction}

1 Dans la littérature scientifique, on peut distinguer au moins deux types d'approches portant sur les dépendances syntaxiques entre constructions. L'une, très majoritaire, aborde la question dans les termes d'un continuum allant de l'intégration à la juxtaposition. L'autre adopte en revanche une vision non-graduelle de l'intégration syntaxique. Ces approches constituent deux réponses distinctes aux limitations liées au caractère par trop rudimentaire des concepts de « subordination » et de « coordination ».

2 Par l'entremise de la comparaison de cadres théoriques qui se sont penchés sur la problématique de l'intégration syntaxique, nous proposons une réflexion sur la « dépendance » grammaticale. Nous esquisserons des pistes pour réaliser l'objectif de se doter d'un concept de « dépendance » univoque et opératoire, en particulier celles-ci : i) une distinction explicite des niveaux de l'analyse linguistique (syntaxe, sémantique, marques morphologiques); ii) une mise au point sur les rapports entre «syntaxe interne » et « syntaxe externe ».

3 Afin de présenter un éventail représentatif de cadres syntaxiques traitant la question des liaisons entre constructions, nous illustrerons, dans un premier temps, l'option 
gradualiste au moyen de la recherche de Raible $(1992,2001)$ d'une part (\$ 1.1.), des travaux de Lehmann (1988) et de Smessaert \& al. (2005) d'autre part (\$ 1.2.). L'option nongradualiste $(\S 2$.) sera représentée par les modèles macro-syntaxiques du Groupe Aixois de Recherche en Syntaxe (Blanche-Benveniste \& al. 1990) et du Groupe de Fribourg (Groupe de Fribourg 2012). Notre premier objectif sera de mettre en lumière les limites des approches graduelles et les apports de la distinction micro- / macro-syntaxe dans ce domaine précis. Notre second objectif sera de confronter les deux approches macrosyntaxiques, avec pour finalité de dégager les implications théoriques pour une modélisation des dépendances syntaxiques. Nous proposerons ensuite quelques pistes de réflexion, en questionnant les rapports entre les niveaux d'analyse, l'extension et la pertinence descriptive du concept de rection (\$3.).

\section{Les modèles gradualistes}

4 Dans l'étude des différents procédés de mise en relation entre constructions, une écrasante majorité d'auteurs font l'hypothèse d'un continuum allant de l'intégration à la juxtaposition. Il en va ainsi, par exemple chez Foley \& Van Valin $(1984: 242)$, de la notion de « cosubordination » qui suggère l'existence d'un continuum entre subordination et coordination.

\subsection{Le modèle de Raible (revisité par Koch et Wiesmath)}

Raible (1992) reprend au travail de Seiler (1988a, b) sur les universaux le concept de dimension, une dimension comportant deux principes opposés (Junktion vs Aggregation). La jonction (Junktion) constituerait une dimension universelle, et chaque langue exploiterait les procédés de jonction selon ses besoins et les contraintes inhérentes à son système ; toutes les langues ont en effet à enchaîner d'une manière ou d'une autre des segments de discours ${ }^{1}$. Raible ventile les «techniques » de jonction sur un continuum situé entre deux principes opposés, la juxtaposition (Aggregation) et l'intégration. Koch résume ainsi la démarche de l'auteur :

Tous les procédés de jonction se situent sur un continuum défini par deux principes fondamentaux : la juxtaposition de deux phrases et l'intégration d'une séquence dans une autre. Ce continuum, qui reflète le caractère plus ou moins 'intégratif' des procédés de jonction, est universel en tant que tel, mais chaque langue l'organise évidemment selon les besoins et les contraintes de son propre système. (Koch 1995 : 15)

Wiesmath (2006) présente les choses comme suit, soulignant également la nature graduelle de l'intégration syntaxique :

Une dimension englobe toutes les options qui se présentent aux locuteurs pour traduire un problème cognitif universel dans le système linguistique. (...) Les différentes stratégies qui se situent le long de cette dimension ou de ce continuum ne se présentent donc pas comme des catégories disjointes, mais comme des transitions graduelles. (Wiesmath $2006: 69-70$ )

7 L'échelle d'intégration reproduite ci-dessous est due à Raible (1992, 2001), mais nous nous fondons aussi sur les travaux de Koch (1995) et de Wiesmath (2006) qui ont adapté ce modèle pour la description syntaxique du français oral (le modèle de Raible étant un modèle typologique); nous leur empruntons également les exemples illustratifs. Voici 
une présentation succincte des huit stratégies (I à VIII, dans l'ordre d'intégration croissant) visant à résoudre le problème de la jonction en français :

- [I] Parataxe asyndétique. Aucun rapport grammatical n'est identifiable entre deux segments juxtaposés; une relation logique implicite (causale, dans 1) fait que les éléments ne sont cependant pas juxtaposés de manière aléatoire :

(1) Il ne pourra pas partir / il est très occupé. [cité par Wiesmath] ${ }^{2}$

- [II] Parataxe à reprise. Un anaphorique du second membre reprend le membre frontal; il y a donc un lien indirect mais explicite entre les deux segments :

(2) Il est très occupé / c'est pourquoi il ne pourra pas partir. [cité par Wiesmath]

- [III] Parataxe syndétique (coordination). Le rapport entre les deux segments, en lien avec la présence du « coordonnant » car, est considéré comme plus étroit :

(3) Il ne pourra pas partir / car il est très occupé. [cité par Wiesmath]

Dans les niveaux (Ebene) I à III, les deux segments sont les constituants d'une unité plus large, alors que dans IV à VII ci-dessous, le second membre est un constituant du premier.

- [IV] Subordination à verbe conjugué (hypotaxe). Une construction à verbe fini est intégrée dans une construction matrice :

(4) il y a des endroits / qui sont bons encore. [oral, dîner, cité par Koch]

(5) Comme il est très occupé / il ne pourra pas partir. [ibid.]

9 La subordination au sens traditionnel n'est pas la modalité de jonction la plus intégrative . Le terme de "subordination » n'est d'ailleurs en général pas utilisé pour désigner les dépendances infra-propositionnelles, mais uniquement pour les relations grammaticales inter-propositionnelles. Ainsi les dépendances où la tête est un Nom ou un adjectif (la crainte qu'il apparaisse; angoissé qu'elle refuse) échappent en général aux filets de la subordination.

- [V] Subordination à verbe non conjugué. Sont concernées les formes participiales et gérondives :

(6) Etant très occupé / il ne pourra pas partir. [cité par Wiesmath]

10 Le caractère non fini de la forme verbale (étant), octroie à cette technique un degré de « nominalité » supérieur à la technique IV.

- [VI] Syntagmes prépositionnels complexes. Les locutions prépositives concernées sont du genre \{ $\grave{a}+N+d e\}$ ou $\{e n+N+d e\}$ (à condition de, à force de, au détriment de, en vertu de, etc.); les propositions infinitives sont affiliées à cette technique VI :

(7) Il ne veut pas partir / pour cause de fatigue. [cité par Wiesmath]

(8) De peur de trop se fatiguer / il ne veut pas partir. [ibid.]

11 Cette stratégie de jonction sanctionne le passage (der Wendepunkt, Raible 1992:18) du domaine verbal au domaine nominal ${ }^{4}:$ " somewhere on the scale there has to be a turning point where the respective techniques lose all their verbal properties, being fully integrated into the realm of nouns instead $»(\text { Raible } 2001: 596)^{5}$.

- [VII] Syntagmes prépositionnels simples ${ }^{6}$ :

(9) Vu son état de fatigue / il ne veut pas partir. [cité par Wiesmath]

- [VIII] «Phrase intégrée». Il s'agit d'un énoncé constitué par le verbe et ses arguments. La phrase intégrée constitue la technique la plus intégrative ${ }^{7}$.

12 Nous allons maintenant nous pencher sur les contributions de Lehmann (1988) et de Smessaert \& al. (2005). Ces auteurs établissent une gamme de critères classificatoires (ou de tests), puis soumettent des constructions syntaxiques à l'examen : plus la construction répond positivement aux critères, plus elle est tenue pour intégrée. 


\subsection{Les modèles de Lehmann et de Smessaert \& al.}

\subsubsection{L'approche de Lehmann (1988)}

Lehmann (1988), dont la taxinomie est bien connue, mène comme Raible une réflexion à finalité typologique, mais il se fonde sur un système à matrice de traits. L'auteur retient six paramètres sémantico-syntaxiques organisés en continuum (1. à 6.) issus de trois oppositions fondamentales (A, B, C) :

\section{A. Autonomie vs intégration}

1. La hiérarchie (la rétrogradation syntaxique) ou l'absence de hiérarchie entre les deux prédications (axe parataxe $\rightarrow$ enchâssement);

2. Le rang morphosyntaxique des unités connectées (axe phrase $\rightarrow$ morphème) ;

\section{B. Expansion vs réduction}

1. La «dépropositionnalisation » de la proposition subordonnée, i.e. la perte des marques personnelles et temporelles (axe domaine verbal $\rightarrow$ domaine nominal) ;

2. La grammaticalisation de la prédication principale, par exemple un phénomène d'auxiliarisation (axe prédicat indépendant $\rightarrow$ opérateur grammatical);

\section{Isolement vs liaison}

1. Le degré de partage des prédications liées, par exemple la présence ou non de relations anaphoriques, de mise en facteur commun d'un élément, de phénomènes de portée, etc. (axe disjonction $\rightarrow$ identité) ;

2. Le caractère plus ou moins explicite du lien entre les prédications (axe syndèse $\rightarrow$ asyndèse).

Le continuum 1., de Lehmann (entre sociation et dependency) correspond peu ou prou à l'ordonnancement des "techniques» de jonction de Raible (entre Aggregation et Integration). Le continuum 2., qui concerne le rang syntaxique des unités, fait écho - en la complexifiant - à la tripartition de Foley \& Van Valin (1984) : periphery, core et nucleus. Le continuum 3., qui a trait à la perte par un constituant propositionnel de ses propriétés verbales (flexion, modes, aspect, force illocutoire), fait penser au passage du domaine verbal au domaine nominal dans le modèle de Raible (dès la technique VI). Le continuum 4., dédié au phénomène diachronique de grammaticalisation et le continuum 5., voué au partage des propriétés modo-aspecto-temporelles et à l'entrelacement des constituants n'entrent pas explicitement dans le système de Raible. Lehmann consacre le continuum 6., à la présence ou à l'absence d'un moyen de connexion entre deux constituants (anaphore, connecteurs), ce qui présente l'avantage de distribuer sur deux axes distincts la problématique de l'étroitesse de la relation syntaxique et celle de la marque morphologique qui pourrait en être l'indice (cf. infra § 3.1.2.). Le modèle raiblesien, qui ne postule qu'un seul axe d'intégration, ne permet pas de distinguer aussi clairement les deux dimensions. De manière globale, ces continua illustreraient la tension entre deux forces opposées, celle de l'élaboration et celle de la compression (Lehmann 1988 : 217); cela rappelle la tension qu'identifie Raible entre agrégation et intégration.

Lehmann prend soin de souligner les corrélations entre ces axes. Ainsi, pour les axes 1 et 2, « advanced hierarchical downgrading of the subordinate clause implies a low syntactic level for it » (ibid., 214), mais l'inverse n'est pas vrai. Le fait qu'une forte intégration d'un 
constituant verbal (axe 1) soit corrélé avec un degré de "verbalité » moindre ( desententialization, axe 3) rejoint les observations de Raible. Une grammaticalisation avancée (axe 4) présuppose une forme de dépropositionnalisation (axe 3) ou un degré de partage élevé des prédications (axe 5). Un lien explicite (axe 6) - anaphore, conjonction est en rapport avec le niveau syntaxique (axe 2) : plus le rang syntaxique est bas, plus les modalités de syndèse sont contraintes.

Un autre intérêt de la contribution de Lehmann est un éclaircissement terminologique. En effet, il essaie de donner un contenu précis à des notions telles que subordination, hypotaxe, enchâssement (embedding), coordination et parataxe. Mais surtout l'auteur (1988: 181) donne une définition du phénomène de dépendance: " $\mathrm{A}$ grammatical relation $\mathrm{R}$ connecting syntagms $\mathrm{X}$ and $\mathrm{Y}$ is a relation of dependency iff $\mathrm{X}$ occupies a grammatical slot of $\mathrm{Y}$ or vice versa. In a dependency relation, $\mathrm{Y}$ depends on $\mathrm{X}$ iff $\mathrm{X}$ determines the grammatical category of the complex and thus its external relations ». Cependant, le cadre proposé n'est pas accompagné de critères utilisables dans toutes les langues, ce qui peut rendre délicate son application à une langue particulière. Smessaert \& al. (2005) se proposent justement d'adapter ce cadre pour la description d'une langue particulière.

\subsubsection{L'approche de Smessaert \& al. (2005)}

17 En adoptant une méthodologie inspirée de l'Approche pronominale (Blanche-Benveniste \& al. 1984), qui est un modèle non graduel, les auteurs (2005: 475) étudient « the degrees of syntactic connectedness between the main clause predicator and the subclause it combines with », à travers l'analyse de 55 « subordinating conjunctions » en néerlandais.

18 La recherche est centrée sur les « subordonnants », pourtant par nature polyfonctionnels, ce que les auteurs (ibid., 509) montrent par ailleurs - telle conjonction pouvant entrer dans plusieurs types de relations. Les auteurs placent les subordonnants sur un continuum allant de l'«endotaxe» à l'«exotaxe», en passant par l'«épitaxe »: " endotactic and exotactic configurations constitute opposite ends of the syntactic continuum, with various epitactic configurations lying in between» (ibid., 498). L'endotaxe est caractérisée par la possibilité qu'une proforme soit proportionnelle à la construction introduite par une conjonction (champ «Propor.» dans le tableau cidessous). Seule la proportionnalité complète est concernée, c'est-à-dire l'équivalence entre la proforme et l'intégralité de la « conjonctive ». La sélection lexicale par un verbe (champ « Sélection » dans le tableau ci-dessous) caractérise les éléments valenciels. En ce qui concerne la proportionnalité interne (« Propor. interne » dans le tableau), conservant tout ou partie de la locution conjonctive, ce critère est utilisé pour faire des distinctions à l'intérieur des constructions épitactiques. Pour un exemple comme :

(10) La décision signifie l'arrêt de toute activité du fait que nous n'avons pas trouvé de repreneur. [ex. inventé]

la proportionnalité interne consiste à déterminer s'il est possible de donner une équivalence sous la forme de ce fait ou de quel fait. Cela permet à Smessaert et al. (2005) de faire la distinction, à l'intérieur des constructions épitactiques, entre les intrinsèques (acceptant la proportionnalité interne) et les extrinsèques (n'acceptant pas la proportionnalité interne). Les auteurs distinguent également deux types de clivage: le clivage « classique » de la conjonctive («Clivage conjonctive» dans le tableau ci-dessous) et le clivage de la conjonctive après transformation par proportionnalité interne (« Clivage propor. interne » dans le tableau ci-dessous). Cela revient à se poser la question 
suivante: si la proportionnalité interne est possible, peut-on la cliver? L'exemple (11) illustre le clivage d'un constituant ayant subi une proportionnalité interne :

(11) ? C'est de quel fait que la décision signifie l'arrêt de toute activité ? [ex. inventé]

Le dernier test consiste à voir si le fait de mettre la conjonctive en tête d'énoncé exige une modification de l'ordre des mots dans la « principale » (« Champ gauche + VSO » dans le tableau ci-dessous) ou si l'ordre reste le même («Champ gauche - VSO » dans le tableau ci-dessous). C'est ce dernier test qui permet de distinguer l'épitaxe de l'exotaxe. L'extraposition sans modification de l'ordre des mots s'accompagne généralement d'une variation des caractéristiques intonatives du segment. Smessaert \& al. (2005) proposent également une sous-catégorisation en lien avec l'implication du locuteur ou sa subjectivité. Les « corrélatives » renvoient à une relation factuelle entre la construction " principale » et la "subordonnée ». Les « commentatives » font intervenir une relation subjective de la part du locuteur. Les « interprétatives » se situent entre ces deux pôles.

Smessaert \& al. (2005) utilisent principalement les critères de l'Approche pronominale avec l'objectif de déterminer des degrés de dépendance. En fonction du nombre et de la nature des critères ayant pour résultat un énoncé grammatical, les auteurs considèrent qu'il s'agit d'une relation plus ou moins étroite. En définitive, ils proposent donc une alternative à la composante macro-syntaxique du modèle aixois (la modularité en moins). Selon eux, il y aurait des relations syntaxiques distinctes entrant dans le cadre d'un continuum. Voici un résumé de leur cadre général sous forme de tableau :

\begin{tabular}{|c|c|c|c|c|c|c|c|c|c|}
\hline & & & Sélection & Propor. & $\begin{array}{l}\text { Clivage } \\
\text { propor. } \\
\text { interne }\end{array}$ & $\begin{array}{l}\text { Clivage } \\
\text { conjonctive }\end{array}$ & $\begin{array}{l}\text { Champ } \\
\text { gauche } \\
+ \text { Vso }\end{array}$ & $\begin{array}{l}\text { Propor. } \\
\text { interne }\end{array}$ & $\begin{array}{l}\text { Champ } \\
\text { gauche } \\
\text { - VSO }\end{array}$ \\
\hline \multirow{2}{*}{ Endotaxe } & Valence & & + & + & + & + & + & + & - \\
\hline & Rection & & - & + & + & + & + & + & - \\
\hline \multirow{6}{*}{ Epitaxe } & \multirow{2}{*}{ Corrélative } & Intrinsèque & - & - & + & + & + & + & - \\
\hline & & Extrinsèque & - & - & - & + & + & - & - \\
\hline & \multirow{2}{*}{ Interprétative } & Intrinsèque & - & - & - & - & + & + & - \\
\hline & & Extrinsèque & - & - & - & - & + & - & - \\
\hline & \multirow{2}{*}{ Commentative } & Intrinsèque & - & - & - & - & - & + & - \\
\hline & & Extrinsèque & - & - & - & - & - & - & - \\
\hline Exotaxe & Illocutive & & - & - & - & - & - & - & + \\
\hline
\end{tabular}

\subsection{Synthèse}

Raible classe des constructions relativement à l'étroitesse de la relation syntaxique en se fondant sur les formes. Le primat de la forme comme critère de décision constitue une approche plutôt traditionnelle. Smessaert \& al. classent des connecteurs (et non des 
constructions) en fonction de la relation syntaxique qu'ils marqueraient (tout en reconnaissant - on l'a dit - la polyfonctionnalité des connecteurs). La classification des connecteurs résulte de l'application de tests morphosyntaxiques (proportionnalité avec une proforme, clivage, etc.). Lehmann classe des constructions en fonction de critères sémantico-syntaxiques. L'approche est d'ordre quantitatif, puisque le degré d'intégration est fonction du nombre de critères satisfaits ${ }^{8}$. On voit que la finalité des théories n'est vraiment pas la même.

Des travaux de haute tenue ont été menés sur le français dans (ou inspirés par) les cadres typologiques de Raible et de Lehmann, ce qui atteste le caractère opératoire de ces taxinomies. On peut citer les travaux de Defrancq (2005), d'Hadermann \& al. (inter alia 2010) et de Pierrard (inter alia Havu \& Pierrard 2008) dans le cadre lehmannien, les recherches de Koch (1995), de Wiesmath (2006) et de Kabatek \& al. (2010), dans le cadre raiblesien. Ces approches ont beaucoup d'avantages: elles sont flexibles (on peut exploiter une partie seulement des continua de Lehmann, par exemple), elles paraissent objectives (Benzitoun 2007) et - aspect non négligeable - elles permettent d'augmenter les connaissances sur la grammaire des langues. Les critères syntactico-sémantiques exploités dans ces travaux sont effectivement très utiles pour la description linguistique (ce qui ne veut pas dire qu'ils le soient pour la saisie du concept de dépendance grammaticale).

\subsection{Evaluation critique des approches gradualistes}

23 Nous nous limiterons à deux points : le recours à la notion de continuum (§ 1.4.1.) ainsi que l'ordre et le nombre des niveaux d'intégration (\$ 1.4.2.).

24 Au moins, les taxinomies étudiées permettent-elles (a priori) de se passer des notions de subordination et de coordination et des apories auxquelles elles mènent (inter alia Deulofeu 1999, Benzitoun 2006, Corminboeuf 2009). En effet, il est important de préciser qu'à l'heure actuelle, de nombreux chercheurs contemporains utilisent encore ces deux notions sans précaution particulière et postulent leur universalité 9 . C'est le cas par exemple de Culicover \& Jackendoff (2005) : l'extrême sophistication de la théorie, voire la virtuosité de l'expertise linguistique tranche avec le caractère sommaire de l'opposition mal dégrossie /subordination vs coordination/ dont ils se servent ${ }^{10}$. Les auteurs convoquent volontiers cette opposition dans leurs travaux sur les relations entre constructions (cf. le titre de chapitre Semantic Subordination despite Syntactic Coordination, dans leur ouvrage de 2005).

Dans le domaine des études sur l'oral, cela fait bien longtemps que cet outillage théorique daté a été abandonné. Et les approches raiblesienne et lehmannienne vont dans cette (bonne) direction.

\subsubsection{Le concept de continuum}

Selon Deulofeu (1986: 80) - qui défend une position opposée aux approches graduelles -, les "effets de gradualité " sont dus "à l'interférence entre relations syntaxiques et propriétés idiosyncrasiques des subordonnants (valeur lexicale et statut morphologique entre autres)»(v. infra $\S 3.1 .2$.). Ils sont sans doute également dus au fait que les catégories notionnelles dont se servent les chercheurs sont elles-mêmes graduelles. C'est le cas par exemple des critères de dépropositionnalisation, de grammaticalisation et de 
celui du degré de partage des prédications de Lehmann. Si les catégories ne sont pas discrètes, qu'elles sont d'emblée organisées en continuum, il est peu étonnant de déceler des transitions graduelles.

Une notion comme celle de transition graduelle demeure partiellement a-théorique. A défaut d'être scientifiquement rigoureuse, la notion de continuum est cependant confortable pour le chercheur. Nul besoin en effet de trancher entre deux analyses, puisqu'il suffit d'introduire des seuils sur le continuum et de placer chaque fait de langue entre deux seuils ${ }^{11}$. En plus de son statut faiblement théorisé, un autre inconvénient est qu'un continuum (même avec des seuils) gomme les différences. On peine à discerner ce qui est différent et ce qui est identique entre deux constructions. Cela peut constituer, entre autres incommodités, un obstacle pour dresser un inventaire des constructions syntaxiques d'une langue, étant donné qu'il n'y aurait quasiment aucune limite au nombre de constructions pouvant être distinguées.

\subsubsection{L'ordre et le nombre des techniques de jonction}

Chez Raible, l'ordonnancement des "techniques» de jonction ainsi que leur nombre appellent plusieurs questions.

- Pour Raible, les syntagmes prépositionnels sont plus intégrés que les constructions verbales introduites par une conjonction (parce que ou comme, par exemple). On peut cependant douter que ce postulat soit généralisable : en fonction de quels critères peut-on dire que des syntagmes prépositionnels comme quant à $\mathrm{x}, \ldots$ de toute façon,... ou à dire vrai,... sont plus intégrés que s'il fait beau dans s'il fait beau, je vais me promener?

- On peut aussi se demander pourquoi une relative serait moins intégrée qu'une participiale? Le segment qui sont bons dans des endroits qui sont bons (4, supra) serait moins intégré que le segment étant très occupé dans étant très occupé, il ne pourra pas partir (6, supra). Il nous semble que l'on pourrait tout aussi bien soutenir le contraire, à savoir qu'une relative est plus intégrée qu'une participiale (notamment lorsque cette dernière est extraposée).

- Les conjonctives (en comme ou en parce que) et les relatives sont rangées dans la même catégorie, alors que leur fonctionnement est manifestement distinct. Une relative entretient une éventuelle relation de dépendance avec un nom, alors qu'une conjonctive l'entretient généralement avec un verbe ; à notre sens, il y a un intérêt à distinguer l'incidence à un nom, de l'incidence à un verbe ou de celle à un énoncé (syntaxe externe).

- A propos du rang syntaxique, peut-on, comme le fait Koch, opposer sur un même plan le rapport qu'entretient une nominalisation avec le reste de l'énoncé et deux constructions verbales dont l'une est en rapport hiérarchique avec l'autre $?^{12}$ Est-ce comparable? Lehmann prend soin de distinguer le niveau syntaxique (continuum 2 dans son modèle) et le rapport hiérarchique (continuum 1 ).

Les principes de regroupement des constructions - pas toujours déclarés - résultent donc de décisions théoriques qui réclament un examen minutieux.

La question du nombre des techniques de jonction chez Raible et d'oppositions polaires chez Lehmann est également un aspect problématique :

- Quels sont les choix qui président à la granularité de l'analyse ? Comment décide-t-on du nombre de niveaux ou d'oppositions distingué(e)s?

- Lehmann et Raible disent de certaines structures binaires qu'elles sont paratactiques, mais sans raffiner l'analyse et sans décrire méthodiquement ces différentes organisations paratactiques. Dans le même esprit, à propos de la technique IV de Raible, d'aucuns 
introduiraient volontiers des sous-catégories. On peut penser à ce titre aux nombreux travaux autour du concept problématique de "subordination » menés par le Groupe de Fribourg et les chercheurs d'Aix-en-Provence. Les cas de figure subsumés sous la technique IV sont très différents, pourtant ils ressortissent à cette seule technique IV. Les techniques VI et VII - qui mettent en jeu des prépositions, simples ou complexes - peuvent sembler très proches, pourtant Raible et Wiesmath en font deux catégories. Selon le point de vue adopté ou la connaissance du domaine par les auteurs, on fera l'économie de sous-catégories ou celles-ci seront au contraire multipliées.

- Comme il s'agit d'un continuum - les techniques se chevauchent ${ }^{13}-$, rien n'empêcherait $a$ priori d'introduire des techniques supplémentaires. Le fait que Koch introduise une technique de plus $\left(\mathrm{IV}^{*}\right)$ pour l'infinitif, témoigne de ce caractère flexible ${ }^{14}$. Tout est question de précision de l'analyse. aussi bien graduelles que non-graduelles - sera abordé plus bas (\$ 2.3.).

\section{Les modèles non-gradualistes : l'exemple des approches macro-syntaxiques}

Pour le paradigme non-gradualiste, nous présenterons les points communs et quelques divergences entre des approches postulant l'existence de deux modes d'organisation du flux discursif, le plan micro-syntaxique et le plan macro-syntaxique : le modèle "modalmodulaire » du Groupe Aixois de recherche en syntaxe (inter alia Blanche-Benveniste \&. al. 1984, 1990) et le modèle « énonciatif-stratificationnel » du Groupe de Fribourg, animé par Berrendonner (inter alia 2002, 2012 ; Groupe de Fribourg 2012) ${ }^{15}$.

Les divergences entre les deux modèles découlent essentiellement du champ d'application assigné au phénomène de rection : acception large pour Fribourg, restreinte pour Aix-en-Provence (pour une discussion, voir Blanche-Benveniste 2012 et Berrendonner 2012).

\subsection{L'approche « modulaire » du Groupe aixois de recherche en syntaxe}

L'approche aixoise, inspirée de l'Approche pronominale (Blanche-Benveniste \& al. 1984), considère que l'articulation entre micro-syntaxe et macro-syntaxe est de type modulaire. Concrètement, cela signifie que tout énoncé donne lieu à des analyses se situant dans les deux modules syntaxiques. Pour un énoncé comme :

(12) Quand les amateurs allaient à la pêche le dimanche matin ils sortaient avec des cannes à pêche partout [exemple oral simplifié, cité par Deulofeu 1991 : 20]

Le statut de l'élément «quand les amateurs... le dimanche » est intéressant parce qu'on peut avoir envie de l'analyser comme porteur des deux types de dépendances : il peut être considéré comme complément de verbe (il répond à la question : quand est-ce qu'ils sortaient ainsi), mais il joue aussi, à un autre niveau, le rôle d'un élément antéposé hors contraste et à intonation ouvrante par rapport à l'ensemble de la construction verbale. (Deulofeu $1991: 20$ )

C'est pour rendre compte de ces deux «types de dépendances » qu'il a paru intéressant aux chercheurs de l'approche aixoise de proposer une analyse modulaire. En outre, il est 
explicitement dit que les différents types de dépendances ne doivent pas être interprétés en termes graduels ${ }^{16}$ :

Des choix théoriques, comme des propriétés de l'objet à décrire, nous conduisent à mettre en évidence deux types de dépendances syntaxiques, fondés sur des principes d'organisation différents, donc de nature totalement différente et entre lesquels nous n'établissons pas de continuité. (Deulofeu 1991 : 19)

La distinction fondamentale se situe entre la rection et l'intégration dans l'énoncé à l'aide d'une relation de dépendance d'une autre nature. La rection inclut la valence et la " simple » rection. La différence entre valence et simple rection n'est pas conçue comme étant d'ordre syntaxique car « La distinction entre les deux domaines, valence et rection, très délicate, est une affaire de lexicographie » (Blanche-Benveniste \& al. $1990: 45)$. C'est la raison pour laquelle la relation primordiale est la rection.

Pour déterminer si un élément est régi ou non, les chercheurs aixois se fondent sur une batterie de tests morphosyntaxiques (proportionnalité avec une proforme, clivage, portée des modalités). Les tests ne sont pas tous placés sur le même plan: celui de proportionnalité est le plus décisif. De plus, certains tests peuvent être neutralisés par la "micro-grammaire des catégories", c'est-à-dire par une distribution propre à une catégorie grammaticale. Les proformes clitiques, par exemple, ne peuvent jamais être clivées, ce qui représente une propriété particulière de cette catégorie grammaticale :

(13) *'est je qui ai fait ça.

En outre, la rection est considérée comme une relation spécifique, la «dépendance » ayant une acception plus large (cf. Blanche-Benveniste 2012:349n). On parlera par exemple de dépendance macro-syntaxique pour des unités comme de toute façon dans de toute façon elle est partie et on en distinguera plusieurs types (infra, § 3.2).

Pour finir, le cadre aixois se définit comme une approche et non comme un modèle ou une théorie. Il n'a pas pour ambition de modéliser la langue de manière systématique mais plus simplement de donner des outils descriptifs aptes à décrire les données langagières, tant orales qu'écrites. En cela, l'approche aixoise est volontairement peu théorisée et est centrée sur la description des données authentiques.

\subsection{Le modèle « stratificationnel » du Groupe de Fribourg}

40 La théorie des unités développée par Berrendonner (1990, 2002, Groupe de Fribourg 2012) repose sur la distinction entre deux types de combinatoires stratifiées, nommées respectivement micro-syntaxe et macro-syntaxe. Dans une approche constructivistecognitiviste du langage, le discours est conçu comme une activité coopérative visant à construire des représentations mentales. Cet ensemble évolutif des représentations publiquement partagées, élaboré coopérativement par les interactants, est appelé mémoire discursive. L'état courant de la mémoire transite d'un stade à un autre, évoluant ainsi à mesure que l'activité interactive se déploie ; en dehors des conduites locutoires et des sous-entendus générés par ces conduites, la mémoire est également alimentée par des connaissances encyclopédiques et par les paramètres de la situation d'énonciation.

41 Dans la macro-syntaxe de Berrendonner, la clause est une unité syntaxique autonome visà-vis de son environnement. Elle consiste en une composition de segments signifiants qui entretiennent des relations de rection (des implications d'occurrence, des contraintes de concaténation, des restrictions sélectionnelles, des accords, etc.). Autrement dit, la clause s'arrête là où s'arrête la rection. 
L'énonciation se définit comme la mise en discours d'une clause (notée $E$ (clause) dans la Figure 1 ci-dessous), ce qui consiste notamment à la pourvoir d'un schème prosodique et d'une instance de prise en charge. Si la clause est un signe, l'énonciation est une conduite actio-verbale qui entretient avec les énonciations adjacentes des relations de type praxéologique. La logique qui préside à l'ordonnancement des énonciations est en effet de nature pragmatique (progression de l'information et planification mémorielle, par exemple). Les énonciations sont des actions communicatives chargées de réaliser des transformations dans la mémoire discursive. Une énonciation, entendue comme une conduite mimo-gestuelle, est donc un opérateur qui s'applique à un état de la mémoire ( $\mathrm{M}$ i) pour en produire un état modifié $\left(\mathrm{M}^{\mathrm{i}+1}\right)$. Le concept de mémoire discursive rend possible l'articulation de deux niveaux distincts de l'analyse linguistique: la syntaxe et la pragmatique. La macro-syntaxe fribourgeoise est ainsi fondamentalement une pragmasyntaxe.

43 Le schéma ci-dessous présente succinctement l'articulation entre micro-syntaxe (morphosyntaxe) et macro-syntaxe (pragma-syntaxe) :

Figure 1 :

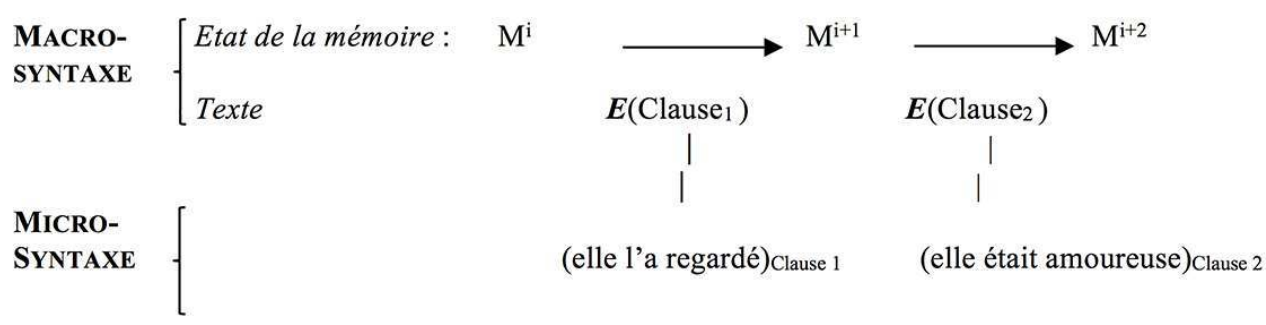

L'articulation entre micro-syntaxe et macro-syntaxe (schéma inspiré de Berrendonner 2002)

L'énoncé oral reproduit dans la Figure 1 (elle l'a regardé elle était amoureuse), recueilli à la volée, signifie qu'il y a eu concomitance temporelle entre le regard de la protagoniste et le sentiment amoureux. Cette relation de concomitance temporelle - qui émerge de l'enchaînement des deux énonciations - est à reconstruire par inférence.

Au plan micro-syntaxique (bas de la Figure 1), la clause 1 n'entretient aucune dépendance formelle avec la clause 2: il n'y a pas de relation micro-syntaxique entre les îlots rectionnels elle l'a regardé et elle était amoureuse (cette analyse serait partagée par les chercheurs du groupe aixois). Au plan macro-syntaxique (haut de la Figure 1), les deux clauses sont actualisées, c'est-à-dire mises en discours (ce que note E(clause)). Les énonciations (de clauses), en opérant successivement sur la mémoire discursive, relient les divers états de celle-ci $\left(\mathrm{M}^{\mathrm{i}}, \mathrm{M}^{\mathrm{i}+1}, \mathrm{M}^{\mathrm{i}+2}\right)$. La suite de deux énonciations elle l'a regardé elle était amoureuse constitue une période binaire.

Un nombre considérable de travaux sur les relations entre constructions ont été menés dans ces deux cadres théoriques. Dans l'approche aixoise, on peut par exemple citer les recherches de Deulofeu (1999), Benzitoun (2006), Sabio (2011), Debaisieux (2008, 2013). Dans l'approche fribourgeoise, on peut citer entre autres les recherches de Corminboeuf (2009), Kallen-Tatarova (2012), Gachet (2012), Avanzi (2012) et la récente Grammaire de la période.

47 Les cadres macro-syntaxiques d'Aix et de Fribourg portent sur le français, ce qui est en soi une originalité, puisque à notre connaissance, une partie non négligeable des travaux qui ont été menés sur les dépendances sont dus à des typologues ${ }^{17}$. Nous pensons que le 
travail minutieux sur les dépendances syntaxiques est non seulement pourvoyeur de connaissances utiles sur les principes combinatoires d'une langue, mais constitue aussi un préalable nécessaire aux généralisations typologiques.

Pour les approches graduelles comme non-graduelles, les critères et les tests convoqués sont parfois délicats à manier, ce que nous allons voir dans la partie suivante.

\subsection{La « crise des critères » 18}

Lorsqu'on veut délimiter des unités (notamment dans un discours oral), ou lorsqu'on veut apprécier le rapport de dépendance entretenu par deux unités, la difficulté principale vient du fait que les indices linguistiques sont généralement peu décisifs.

Voyons par exemple ce qui, pour Raible et ses épigones, constitue un indice d'intégration :

- La présence d'une conjonction ou d'un anaphorique. Or on sait qu'on ne peut pas se fier sans examen aux «conjonctions », fondamentalement polyfonctionnelles, pour déterminer le statut d'une relation syntaxique. Quant à l'anaphore (hors cas de liage), qui a souvent trait à la cohérence macro-syntaxique, elle est généralement de peu de secours pour l'examen des rapports de dépendance grammaticale.

- Le rang syntaxique : plus les constituants sont de bas rang, plus l'intégration est forte (ce critère est mentionné notamment par Foley \& Van Valin 1984 et Lehmann 1988). Là également, est-ce généralisable ? La relation syntaxique entre les constituants de bas rang une girafe un lac un arbre dans Pierre a dessiné une girafe, un lac, un arbre est-elle plus " étroite » que parce que Marie me l'a demandé dans Je suis venu parce que Marie me l'a demandé?

- Une absence de flexion: "l'intégration est d'autant plus forte que les marques du verbe conjugué sont réduites » (Wiesmath $2006: 72 n)^{19}$.

- Un degré de verbalité moindre (corollairement, un degré de nominalité élevé) : "Plus une proposition est intégrée, plus le verbe perd ses caractéristiques verbales » (Wiesmath 2006 : 79).

51 On pourrait encore citer les phénomènes de portée (Haiman \& Thompson 1984), les similitudes des formes verbales des prédications liées, le mode subjonctif souvent considéré comme un indice de subordination, etc.

Un des problèmes auquel on est confronté avec ces critères réside dans leur proximité. En effet, l'absence de flexion, le degré de verbalité (dépropositionnalisation) et le rang syntaxique sont liés (cf. les corrélations entre continua observées par Lehmann) ; il s'agit de critères proches pouvant parfois être dérivés les uns des autres. Un second problème est qu'il faudrait démontrer que la syntaxe externe a une influence sur la syntaxe interne ${ }^{20}$ et donc qu'il est possible de calculer la relation externe à partir d'une description interne du syntagme. Or, à notre connaissance, ce point n'a jamais fait l'objet d'une étude approfondie (voir tout de même la notion de "main clause phenomena » dans Green 1976). Pourquoi devrait-on considérer que pour vivre et pour sa survie sont plus intégrés que pour qu'il vive du seul fait que l'un comporte un verbe à l'infinitif ou un syntagme nominal et l'autre un verbe conjugué ?

Les approches macro-syntaxiques convoquent, quant à elles, surtout deux tests clés pour déterminer si une séquence est régie ou pas :

- La non-autonomie des séquences (dans le modèle fribourgeois): si une séquence morphosyntaxique B implique la cooccurrence d'une autre séquence A, alors B est régie par 
A. Mais le critère n'est pas toujours évident à manier (Gachet 2012, Blanche-Benveniste 2012).

- Le test de proportionnalité à une proforme (dans le modèle aixois). Ce test met en évidence l'existence d'un paradigme d'éléments équivalents à la proforme et donc de contraintes imposées par un élément $\mathrm{A}$ (le recteur) à un élément $\mathrm{B}$ (le régi). Le lien de rection est défini principalement par ce critère. La composition interne de l'unité régie (syntaxe interne) n'est pas prise en compte pour déterminer la nature de la relation externe (mais nous verrons plus loin que l'observation de la syntaxe interne peut constituer une piste intéressante pour aider à la délimitation des dépendances macro-syntaxiques). Ainsi, qu'il s'agisse d'une construction verbale, d'un syntagme prépositionnel ou nominal, la rection sera réputée de même nature, tout comme dans le modèle fribourgeois. Il peut en outre exister des cas difficiles à trancher (cf. Groupe de Fribourg 2012 ; travaux sur la rection faible, par exemple Blanche-Benveniste \& Willems 2007).

Force est de constater que pris isolément, aucun indice segmental n'est déterministe. Il y a dès lors deux échappatoires :

(i) on s'en remet exclusivement à des tests syntaxiques, ou

(ii) on emprunte des critères aux autres niveaux de l'analyse linguistique.

Si on s'en remet aux tests (i), une partie du problème est de savoir ce que ceux-ci montrent réellement (certains tests sont aussi des constructions syntaxiques, par exemple le clivage). Pourquoi une propriété donnée acquiert-elle le statut de critère définitoire? Qu'est-ce qui détermine une réponse négative à un test: une propriété grammaticale ou une contrainte sémantique? On sait par ailleurs que les tests peuvent être neutralisés pour certaines constructions (Deulofeu 2007). Ainsi, lorsqu'un test donné a pour résultat un énoncé agrammatical, les raisons peuvent en être multiples. C'est le cas du clivage dans l'exemple suivant repris de Benzitoun (2007:129) :

(14) Il me regardait comme s'il ne me comprenait pas.

(14') ? C'est comme s'il ne me comprenait pas qu'il me regardait.

Cela met-il en évidence une relation "moins étroite», une neutralisation du test du clivage pour les constructions introduites par comme si ou un autre paramètre? Il se produit un phénomène identique avec pourquoi :

(15) ?C'est pourquoi qu'il a fait ça ?

Et les divergences quant à l'acceptabilité de certains énoncés après application des tests (et donc les variations interindividuelles) sont loin d'être négligeables.

On peut également avoir des conceptions radicalement différentes sur ce que les tests sont censés démontrer. Alors que l'approche aixoise met au centre de ses analyses les critères de clivage, de portée de la négation et de proportionnalité à une proforme, Berrendonner en a une toute autre conception :

Les tests couramment utilisés pour déceler un régime de verbe (possibilité d'être clivé, d'être sous la portée de la négation, de commuter avec des proformes interrogatives, etc.), permettent en fait d'identifier non les éléments régis, mais les éléments rhématisables. Les deux coïncident à peu près dans le cas des rapports $[\mathrm{V}$ $<=\mathrm{A}]^{21}$, mais pas dans les autres domaines de la rection. (Berrendonner $2012: 90$ )

En outre, tous les linguistes ne militent pas en faveur de l'usage des tests pour mener une analyse syntaxique. La principale critique émise à leur encontre réside dans leur caractère définitoire :

Il en va ainsi des tests morpho-syntaxiques dont on use encore trop souvent de manière définitoire plutôt qu'heuristique. Ce n'est pas forcément parce qu'elle ne 
passe pas tel test qu'une structure n'en possède pas la propriété testée : il se peut également que le test ne soit pas fiable hors heuristique ou simplement pas transparent. (Van Raemdonck 2008 : 11) et qu'envers eux une attitude critique est indispensable. Et la manière d'agencer les tests est également un objet de débat. Comme on l'a vu, Smessaert \& al. (2005), qui s'inscrivent pourtant dans le paradigme de l'Approche pronominale, considèrent que plus il y a de critères vérifiés, plus la relation syntaxique est étroite. Blanche-Benveniste \& al. (1990), quant à eux, utilisent les tests comme des indices de l'existence d'une relation de rection et lorsqu'un test particulier ne se vérifie pas, ils l'imputent généralement à un blocage du constituant analysé.

61 Si on emprunte des propriétés aux autres niveaux linguistiques (ii), un problème est évidemment de déterminer si les critères qui fondent la reconnaissance des unités formelles et des rapports que celles-ci entretiennent sont fiables et scientifiquement univoques. Et dans quelle mesure un critère d'ordre sémantique peut-il être révélateur d'une contrainte grammaticale ( $\$ 3.1 .1$. infra) ? On comprend cependant que Lehmann s'appuie sur un assortiment de critères, en faisant l'hypothèse que leur croisement soit une procédure heuristique plus robuste.

\section{Perspectives}

\subsection{Une articulation explicite des niveaux d'analyse ${ }^{22}$}

\subsubsection{Le rapport syntaxe-sémantique}

L'analyse par matrice de critères (par exemple Lehmann) prête le flanc à la critique, dans la mesure où elle ambitionne la saisie d'une relation syntaxique au moyen de contraintes tant lexicales que sémantiques ou pragmatiques (i.e. des contraintes qui ressortissent à des niveaux distincts de l'analyse linguistique), sans les articuler de manière explicite.

Pour apprécier les rapports de dépendance grammaticale, peut-on cependant s'en tenir uniquement au plan syntaxique, en évacuant par principe les critères sémantiques ? Cela paraît plus salubre méthodologiquement mais - même dans les modèles articulant microet macro-syntaxe - la dimension lexico-sémantique est largement présente. Ainsi, la notion de rection de Berrendonner possède une dimension sémantique comme celle de valence chez Blanche-Benveniste (considérée comme une sous-partie de la rection).

- Pour le Groupe aixois de recherche en syntaxe, la valence est fondée sur la sélection lexicale. Plus généralement, on peut dire qu'une partie du sens, à la fois lexical et grammatical, est donnée par l'organisation valencielle et rectionnelle. Lorsque l'on se demande si un constituant est sélectionné par un verbe, il s'agit donc de toute évidence d'un critère lexical et non d'un critère purement syntaxique ${ }^{23}$.

- Pour sa part, Berrendonner (2012) postule qu'une structure de rection élémentaire comprend deux niveaux, l'un syntaxique, l'autre sémantique. A une co-occurrence formelle correspondrait, au plan sémantique, une contrainte de sélection. Ainsi, dans Pierre nuit à son entourage ou ses biens proviennent d'un héritage, les prépositions à et $d(e)$ relèvent de la sélection sémantique, mais on les interprète comme des marques de dépendance. Le produit de la sélection sémantique fonctionne comme une marque de dépendance. Dans cette perspective, il est important de comprendre comment s'articulent dépendances formelles et 
contraintes de sélection mutuelle - c'est-à-dire de formuler explicitement les correspondances entre les deux niveaux d'analyse (non isomorphes). et de rendre explicites les relations entre les niveaux.

\subsubsection{Le rapport entre marqueur morphologique et relation syntaxique}

Quelle est l'influence des marqueurs morphologiques sur la relation syntaxique? Quel type de codification est opéré par les formes? Dans la littérature scientifique, la réflexion porte surtout sur le rapport syntaxe - sémantique, mais plus rarement sur la façon dont syntaxe et marqueurs morphologiques interagissent. Certains travaux ont pourtant montré l'importance de traiter indépendamment les marques morphologiques et la syntaxe :

Une précaution à prendre pour ne pas commettre des erreurs d'analyse est de ne pas établir un parallélisme entre marqueurs morphologiques et relations syntaxiques. Ainsi la présence d'un morphème tel que parce que n'implique pas que la séquence qu'il introduit soit nécessairement subordonnée au verbe principal. Parce que peut en effet marquer, comme beaucoup d'autres conjonctions dites « de subordination » une relation dont les propriétés sont en fait plus proches de celles de la coordination. (...) il faut se donner une définition des relations syntaxiques indépendante des morphèmes qui les marquent de façon plus ou moins stable. (Deulofeu 2001 : 104)

Le processus relationnel doit être distingué du marquage morphologique de cette relation. Ce n'est pas parce qu'une construction comporte le connecteur quand qu'il y a corollairement une relation de dépendance grammaticale. Et une dépendance grammaticale n'est pas forcément marquée par un connecteur. Cela nous conduit à un questionnement central, celui du marquage des relations de rection. Dans ce domaine, il nous semble que l'on peut distinguer deux positionnements théoriques.

i) Une première position, radicale, postule que les relations de dépendance ne sont pas marquées (c'est en quelque sorte l'inverse de la position de Raible) :

(...) il est hautement improbable qu'il existe en français (...) des éléments chargés

d'établir un rapport syntaxique entre deux propositions. Les rapports syntaxiques entre propositions ne résultent pas de la présence de certains éléments, comme que, mais des propriétés lexicales et morphosyntaxiques des éléments qui les composent. (Defrancq $2005: 65$ )

Dans cette perspective, la présence ou l'absence d'un élément comme que ne change pas la nature de la relation.

Mais les chercheurs ont vu depuis longtemps un intérêt à distinguer, au plan syntaxique, les cas de figure suivants :

(16) Vous n'aurez pas ma fille, parce que vous n'êtes point gentilhomme.

(17) Vous n'aurez pas ma fille, car vous n'êtes point gentilhomme.

(18) Puisque vous n'êtes point gentilhomme, vous n'aurez pas ma fille.

(19) Vous n'êtes point gentilhomme, vous n'aurez pas ma fille. [Molière, Le bourgeois gentilhomme]

Si on fait une analyse syntaxique différente de ces quatre énoncés, c'est que la présence ou l'absence du marqueur a un rôle à jouer, tout comme le type de marqueur (car vs parce que vs puisque). L'absence de connecteur induit ici une analyse (non rectionnelle), alors que la présence ne permet pas de décider autrement qu'au cas par cas. 
71 ii) Une seconde position postule que les relations de dépendance peuvent être marquées morphologiquement. Ce postulat conduit à distinguer quatre cas (Berrendonner 2008) :

72 - Relations non marquées: dans je te lègue ma thèse, le régime ma thèse n'est pas marqué comme entretenant cette relation avec le verbe lègue.

73 - Relations marquées sur l'élément régi : les prépositions casuelles comme à dans à mon fils, je lègue ma thèse fonctionnent comme des répliques de relations argumentales imposées par le verbe.

74 - Relations marquées sur l'élément recteur : les clitiques comme lui dans mon fils, je lui lègue ma thèse répliquent des traits caractéristiques de l'argument.

75 - Relations marquées et sur le dépendant et sur la tête : à mon fils, je lui lègue ma thèse.

76 Si on tient les accords - codés morphologiquement - comme relevant de la rection (Berrendonner ibid.), il y a manifestement marquage de certains types de rection. Dans l'hypothèse où certaines organisations rectionnelles sont marquées morphologiquement, on peut se demander - c'est un questionnement essentiel - jusqu'où il convient d'étendre le champ des marqueurs de dépendance, et ce qu'on entend par «marqueur de dépendance »? Par exemple, est-ce que moins dans Moins il parle, moins il m'énerve est un marqueur de dépendance syntaxique, au même titre qu'un clitique ou une préposition casuelle?

77 On voit le nœud du problème : il y a lieu de tenir compte des formes - ce qui semble évident lorsque l'on fait de la syntaxe - sans toutefois leur assigner un rôle univoque et transparent de marqueur de dépendance. Une position qui nous paraît raisonnable serait de dire qu'il peut y avoir des indices de la dépendance sous la forme de marques. Mais une même marque peut induire différentes relations voire même une absence de relation. Par exemple, la préposition pour peut marquer une relation de rection dans certains énoncés, mais pas dans tous. Ainsi dans (20) le constituant introduit par pour est régi, alors que dans (21) il est non-régi :

(20) je connais des producteurs qui ont abandonné les arbres pour revenir à la vigne [oral, CRFP]

(21) ah bon et ouais mais pour revenir à ton histoire de pingouins ça ça se finit comment est-ce que les hommes disparaissent à la fin [oral, Corpaix]

78 Une option théorique possible pourrait être de remplacer la notion de «marqueur de dépendance » par celle de « contrainte ». Une contrainte est un indice formel non décisif pour identifier les relations syntaxiques. Cette option permettrait de tenir compte de certaines formes (par exemple les «conjonctions») sans les considérer comme des preuves de dépendance syntaxique. Elle fait écho à la position de Deulofeu (2001) (voir cidessus) et à celle d'Allaire (1982 : 42) qui postulait que « la conjonction que n'est pas plus 'subordonnante' que et n'est 'coordonnant'« .

\subsubsection{Le rapport entre syntaxe externe et syntaxe interne}

79 Notre approche généralise le positionnement d'Allaire à toutes les marques morphosyntaxiques réputées démontrer une relation syntaxique (subjonctif, mobilité, forme verbale, reprise anaphorique...).

80 Dans les exemples (20-21), la forme interne, très proche, ne permet pas de tirer de conclusion quant au statut syntaxique externe des pour-C. Pourtant, la syntaxe externe est clairement différente, ce que l'on peut montrer grâce à des contraintes non 
observables directement dans la structure lexico-syntaxique réalisée. Dans (20), le segment en pour peut être pronominalisé (en pour ça) et clivé alors que dans (21) l'équivalence avec pour ça est exclue de même que le clivage.

81 Dans le domaine de l'étude des relations entre constructions, une réponse possible serait donc de s'en tenir aux contraintes externes en les articulant aux éventuelles contraintes internes et ainsi de rendre explicite l'influence présumée des premières sur les secondes. Comme nous l'avons montré au sujet des approches graduelles, les indices formels et les critères convoqués sont souvent de type "contrainte interne». Or, il faudrait au préalable étudier de manière systématique s'il existe un impact des relations externes sur la forme interne des constituants, ce qui d'après les exemples que nous avons étudiés jusqu'à présent ne semble pas être le cas (excepté pour les « main clause phenomena » qui sont le résultat d'une absence totale de contrainte).

82 L'explication de cette différence de comportement vis-à-vis des tests qu'avancent les approches des groupes aixois et fribourgeois consiste à postuler pour (20-21) deux organisations syntaxiques radicalement distinctes. De plus, (20-21) présentent d'autres contraintes externes permettant d'étayer notre analyse. En effet, dans (20), la pour-C est enchâssée dans une relative, ce qui semble difficile pour la pour-C de (21). Et inversement, la modalité interrogative de ça se finit comment, qui ne porte pas sur la pour-C dans (21), nous semble réservée à ce type d'emploi non-régi.

\subsection{Plusieurs types de dépendance?}

L'opposition binaire postulée entre micro- et macro-syntaxe n'empêche pas qu'il y ait différents types de relations qui relèvent de l'organisation rectionnelle. De notre point de vue, il existe une seule relation de rection, mais celle-ci se manifeste de diverses façons. Et s'il existe différentes structures de rection (Corminboeuf 2013), cela n'implique nullement que celles-ci soient organisées en continuum.

84 Ainsi, dans le modèle "fribourgeois ", la rection unilatérale où un segment implique la cooccurrence d'un autre (mais non réciproquement) est distinguée de la rection bilatérale où deux segments s'impliquent mutuellement. C'est ce que Hjelmslev (1971, 1968 : 38) appelait respectivement relations de détermination et de solidarité.

Dans le modèle aixois, on distingue aussi communément la valence (fondée sur des contraintes lexicales) de la « simple » rection (fondée sur des contraintes catégorielles). Il faut toutefois noter que le domaine de la rection dans l'approche fribourgeoise est plus vaste que dans l'approche aixoise. En effet, les principaux tests de rection (version aixoise) s'appliquent à des recteurs verbaux alors que ceux utilisés dans l'approche fribourgeoise sont valables pour une multitude de catégories. De plus, dans le cadre du Groupe de Fribourg, les « compléments extra-prédicatifs » (à mon avis, sans doute, selon lui, par exemple) et les constructions introduites par puisque ou quoique relèvent de la rection. Dans l'approche aixoise, on considère toutes ces unités comme étant non-régies, du fait qu'elles ne sont pas proportionnelles à une proforme, pas focalisables et hors modalités.

A notre sens, on gagnerait également à distinguer - en suivant Smessaert \& al. (2005) - les relations d'ordre paradigmatique des relations d'ordre syntagmatique: «a fundamental distinction needs to be made between the (paradigmatic) criterion of proportionality on the one hand, and the two (syntagmatic) criteria of clefting and fronting on the other hand» (Smessaert \& al. 2005 : 512). Les relations paradigmatiques sont mises en évidence 
par le critère de proportionnalité à une proforme, et les relations syntagmatiques sont basées sur le critère d'implication (d'occurrences), avec une vaste zone de recouvrement entre les deux. En effet, paradigmatique et syntagmatique vont très souvent de pair, mais les deux types de critères ne sont pas applicables à tous les contextes et il arrive que deux unités faisant partie d'un même paradigme forment deux syntagmes différents et inversement. C'est le cas notamment pour les exemples en ainsi que étudiés par Benzitoun (2007) :

(22) Je tiens avant tout à en remercier les entreprises ainsi que les investisseurs institutionnels qui ont rejoint notre capital.

Il est clair que les entreprises et les investisseurs institutionnels qui ont rejoint notre capital appartiennent au même paradigme de complément du verbe remercier. En revanche, la pronominalisation d'un des deux éléments composant la liste montre qu'il s'agit de deux syntagmes distincts :

(23) Je tiens avant tout à les en remercier ainsi que les investisseurs institutionnels

qui ont rejoint notre capital. [internet]

Au plan macro-syntaxique aussi, il y a lieu de distinguer plusieurs types de dépendances. On a vu que Lehmann et Raible disent de certaines relations entre constructions que celles-ci sont de nature paratactique, mais il conviendrait de raffiner l'analyse et de décrire méthodiquement les diverses organisations (Béguelin, Avanzi \& Corminboeuf 2010). Par exemple une construction temporelle comme je suis arrivé il était vingt heures présente des propriétés très différentes d'une construction également à interprétation temporelle comme elle l'a regardé elle était amoureuse (Benzitoun 2010). Dans cet esprit, les chercheurs d'Aix font la distinction entre différents types de dépendance macrosyntaxiques (Debaisieux 2013). Dans les exemples ci-dessous, les deux constructions en quand ne sont pas proportionnelles à la proforme quand. En cela, elles ne sont pas régies. Cependant, la première est mobile alors que la seconde est bloquée en postposition, ce qui suggère qu'il s'agit de deux organisations macro-syntaxiques différentes :

(24) c'est pas affreux mais enfin quand on y pense il y a de quoi rire [oral, Corpaix]

(25) Frédéric marchait sur la route, quand tout à coup une sentinelle croisa la

baïonnette. [Flaubert, L'éducation sentimentale]

89 Le Groupe de Fribourg (2012) a de son côté proposé une modélisation de plusieurs «routines praxéologiques» en français. La notion de "routine» pose d'emblée la question d'une forme de dépendance macro-syntaxique.

\subsection{L'intérêt à distinguer rection et absence de rection}

90 On peut se demander quel est le gain descriptif obtenu en mesurant la force de la dépendance (dans les modèles typologiques de Raible et Lehmann), et réciproquement, le gain obtenu en distinguant les segments qui sont régis de ceux qui ne le sont pas (dans les cadres aixois et fribourgeois). Une critériologie comme celle de Lehmann vise à jauger la force de la dépendance; la batterie de critères classificatoires mobilisée permet d'apprécier l'intensité de la relation, qui est fonction du nombre de propriétés satisfaites. La méthode comparative adoptée par les modèles graduels (par exemple telle structure est dite plus intégrée que telle autre) conduit donc à ne pas décider si une construction est rectionnellement connexe ou non. Par exemple, dans un énoncé comme Entre nous, il y a un problème entre eux, le complément périphérique entre nous sera apprécié comme moins intégré que le complément entre eux, ce qui correspond bien à l'intuition (et 
encore, nous ne sommes pas certains que les cadres en question puissent saisir cette différence). Mais la question est aussi de savoir si entre nous est grammaticalement dépendant du verbe avoir ou non, information à laquelle les modèles gradualistes ne donnent pas accès (précisément parce qu'ils sont gradualistes). Autrement dit, il n'est pas postulé d'opposition entre rection et non-rection. Il en découle une fois de plus que les objectifs théoriques et descriptifs sont sensiblement différents : on ne prétend pas saisir la même réalité que dans les modèles non-gradualistes.

91 En quoi cette opposition entre rection et absence de rection est-elle si essentielle, pour les modèles non-gradualistes?

92 Pour le Groupe de Fribourg, cette distinction est cruciale. Un seuil fonctionnel est postulé entre la micro-syntaxe (qui correspond au champ d'action de la rection) et la macrosyntaxe, i.e. ce qui excède la syntaxe de rection. Passé ce seuil, les dépendances observables changent radicalement de nature. Autrement dit, il ne peut en aucun cas y avoir un continuum. Au changement d'unités correspond un changement de fonctionnalité, la macro-syntaxe étant conçue comme une troisième articulation du langage (au sens de Martinet). L'hypothèse fondamentale est que les discours monologaux résultent de la combinaison de deux ordres de combinatoires superposés et irréductibles l'un à l'autre: une combinatoire de signes (micro-syntaxe) et une combinatoire d'actions communicatives (macro-syntaxe). Une clause est un signe complexe remplissant une fonction significative, alors qu'une énonciation de clause est une conduite actio-verbale à fonction communicative; du point de vue sémiotique, c'est donc très différent. Cela explique pourquoi il est si important de pouvoir identifier les relations de rection.

93 Cerner le concept de rection est nécessaire pour le découpage du discours en clauses ( = des îlots rectionnels), qui est elle-même une étape essentielle de l'analyse linguistique, dans la mesure où une énonciation ( = la mise en discours d'une clause) est, dans le modèle macro-syntaxique, un opérateur sur la mémoire discursive ( = l'ensemble évolutif des représentations publiquement partagées). Une structure binaire qui forme une seule énonciation (une minute plus tard, le train déraillait, cf. Corminboeuf 2010) a ainsi des effets cognitifs très différents d'une structure binaire qui consiste en deux énonciations, donc qui opère deux fois successivement sur la mémoire discursive (une minute de plus et le train déraillait).

Pour le cadre aixois, la distinction entre rection et non-rection est également fondamentale. C'est ce qui permet de distinguer "les constructions, domaine où s'exercent les rections » et "les énoncés, domaine des relations macro-syntaxiques " (Deulofeu 1991: 20). Cela donne lieu à deux modes d'organisation syntaxique bien distincts : d'un côté les éléments intégrés à un paradigme (virtuel ou pas) et d'un autre côté les éléments s'insérant dans un énoncé hors de tout paradigme. Cependant, cela ne recoupe pas exactement la distinction entre micro- et macro-syntaxe (contrairement à l'approche fribourgeoise). Si les unités non-régies ne peuvent donner lieu qu'à une analyse macro-syntaxique ( $\mathrm{du}$ moins en ce qui concerne leur syntaxe externe), les relations entre unités régies sont décrites au moins du point de vue de la micro-syntaxe mais elles peuvent également avoir une contrepartie macro-syntaxique. C'est le cas pour ce que les chercheurs aixois appellent les dispositifs de la rection verbale et l'insertion d'un élément régi dans le champ gauche de l'énoncé.

De plus, les unités non-régies sont décrites en termes de configuration, d'effets de cohésion, d'interdépendance, d'absence d'autonomie et de contour prosodique, ce qui 
peut donner lieu à des analyses et des interprétations différentes. Ce qui est mis plus particulièrement en avant dans le domaine de la macro-syntaxe, ce sont les contraintes sur les modalités. En effet, lorsque l'on bascule dans la macro-syntaxe, l'élément portant les modalités aura le statut d'élément central (le noyau) et les autres éléments hors modalités et ne pouvant pas avoir leur modalité propre le statut d'éléments périphériques (les ad-noyaux).

L'attention portée à la saisie des dépendances syntaxiques est en quelque sorte constamment d'actualité, même si à ce propos la linguistique ne parle pas d'une même voix $^{24}$. Cela est dû au développement des études sur l'oral et aux problèmes de segmentation rencontrés. En effet, lorsque l'on souhaite procéder à l'annotation syntaxique d'une transcription, il est indispensable de délimiter une unité syntaxique maximale dans laquelle toutes les parties sont reliées par des rapports de dépendance. La définition de cette unité fait actuellement l'objet de recherches (Benzitoun \& al. 2010).

L'intérêt pour les dépendances syntaxiques est également - et sans doute principalement - dû aux études typologiques (cf. Raible 1992, Lehmann 1988, Rebuschi 2001-2002, etc.). La reconnaissance, la description fine et la représentation réelle des structures de dépendance permettent de mieux situer une langue donnée parmi les autres langues; on peut penser à l'opposition entre langues "concentriques » et langues «excentriques ", opposition fondée sur la topographie du marquage de la dépendance - sur la tête ou sur le dépendant (Milewski 1967). Nichols (1986) montre non seulement que la connaissance des structures de rection d'une langue constitue un axe typologique, mais que cette opposition "dependent-marking " vs « head-marking " constitue également une entrée privilégiée sur la diachronie des langues. Etudier les structures de rection et les différents types de dépendance permet de mieux connaître les structures syntaxiques d'une langue et de construire des points de comparaison avec d'autres langues. La formulation de régularités formelles requiert un concept de dépendance explicite et scientifiquement établi.

Enfin, c'est une tâche ordinaire de la description linguistique que de définir la nature des relations de dépendance et de dégager les principes combinatoires qui gouvernent ces relations. (Mais cela dépend bien sûr du rôle que l'on assigne à la grammaire). Tout cela justifie l'intérêt des linguistes pour la problématique de la dépendance grammaticale.

Si un grand nombre de travaux sur les dépendances - et non des moindres - ne définissent pas le concept de dépendance, c'est qu'ils n'en ont manifestement pas besoin pour remplir les objectifs qu'ils se fixent. En effet, la définition de "dépendance » ou « intégration »- voire de "relation syntaxique »- est rarement explicite alors qu'elle est pourtant centrale pour la recherche en syntaxe. Peu nombreux sont les cadres théoriques qui questionnent ou qui définissent la notion de dépendance. Il nous parait important de définir la notion, quitte à ce que l'extension de celle-ci soit différente d'un cadre théorique à l'autre.

\section{Conclusion}

La littérature scientifique dans le domaine des dépendances syntaxiques est, on s'en doute, particulièrement abondante. La plupart des approches assument le présupposé selon lequel les relations syntaxiques externes sont marquées par des indices formels réalisés dans la structure interne (conjonctions, prépositions, forme verbale, degré de 
nominalité, etc.). Une conséquence directe de ce postulat est l'adoption d'une conception graduelle de l'intégration syntaxique, dont nous avons souligné les limites.

Nous avons également montré que l'on peut faire deux analyses syntaxiques radicalement différentes de segments qui sont en apparence très proches (ex. 20-21). Cela nous a conduits à proposer une alternative à cette corrélation entre "marqueur " et relation syntaxique pour décrire les liens entre constructions. Le concept de «contrainte » nous paraît plus fécond que celui de "marqueur de dépendance» étant donné que les marqueurs comme les conjonctions sont d'une part polyfonctionnels et que, d'autre part, ils peuvent être absents (cas de relations entre constructions non-marquées). L'identification des contraintes peut se faire au moyen de tests syntaxiques (manipulations) ou par l'observation d'indices présents dans la structure des énoncés (enchâssement dans une relative vs modalité interrogative dans 20-21). L'analyse gagnerait à ne pas être centrée sur un seul indice ou sur un test mais plutôt sur un faisceau d'indices concordants. Cela revient à adopter une conception globale plutôt que locale de l'exploitation des indices formels pour l'analyse linguistique et à n'assigner a priori à aucun indice le statut de marqueur de dépendance.

Les cadres qui adoptent une distinction entre micro- et macro-syntaxe ne postulent $\mathrm{ni}$ lien nécessaire entre "marqueur» et relation syntaxique, ni degrés d'intégration syntaxique. En cela, ils nous paraissent actuellement les mieux à même de résoudre certains des problèmes soulevés par les relations entre constructions et de nourrir une réflexion sur les dépendances syntaxiques.

\section{BIBLIOGRAPHIE}

Allaire, S. (1982). Le modèle syntaxique des systèmes corrélatifs. Thèse d'État, Université Rennes 2, Lille, ANRT.

Avanzi, M. (2012). L'interface prosodie / syntaxe en français. Dislocations, incises et asyndètes. Bruxelles, Peter Lang.

Béguelin, M.-J., Avanzi, M., Corminboeuf G. (éds). La parataxe, 2 volumes. Berne, P. Lang.

Benzitoun, C. (2006). Description morphosyntaxique du mot quand en français contemporain. Thèse de Linguistique Française, Université de Provence.

Benzitoun, C. (2007). « Approche comparative de la notion de degré en syntaxe à travers l'opposition entre subordination et coordination ». Travaux de linguistique 54-1. 121-132.

Benzitoun, C. (2010). Comment tirer profit de la parataxe ? Etude sur les enchaînements de constructions verbales. In M.-J. Béguelin, M. Avanzi, G. Corminboeuf, La Parataxe : Entre dépendance et intégration syntaxique, Tome 1, Collection Sciences pour la communication 91. Berne, Peter Lang. 153-174.

Benzitoun, C. \& Sabio, F., (2010). « Où finit la phrase ? Où commence le texte ? », Discours 7, http://discours.revues.org/7966. 
Benzitoun, C., Dister, A., Gerdes, K., Kahane, S., Pietrandrea, P., Sabio, F., Debaisieux, J.-M. (2010). « tu veux couper là faut dire pourquoi. Propositions pour une segmentation syntaxique du français parlé ». In F. Neveu, V. Muni Toke, J. Durand, T. Klingler, L. Mondada, \& S. Prévost (éds.), Congrès Mondial de Linguistique Française - CMLF 2010. 2075-2090.

Berrendonner, A., (1990). « Pour une macro-syntaxe ». Travaux de linguistique 21. 25-36.

Berrendonner, A., (2002). « Les deux syntaxes ». Verbum XXIV. 23-35.

Berrendonner A. (2008). « Dislocation et conjugaison en français contemporain ». Cahiers de praxématique 48. 85-100. http://praxematique.revues.org/779

Berrendonner, A., (2012). « Autour de la rection ». In S. Caddéo, M.-N. Roubaud, M. Rouquier \& F. Sabio (éds), Penser les langues avec Claire Blanche-Benveniste. Aix-en-Provence, Publications de l’Université de Provence. 83-91.

Blanche-Benveniste, C., (1981). « La complémentation verbale : valence, rection et associés ». Recherches sur le français parlé 3. 57-98.

Blanche-Benveniste, C., (2003). « Le recouvrement de la syntaxe et de la macro-syntaxe ». In A. Scarano (éd.), Macro-syntaxe et pragmatique. L'analyse linguistique de l'oral. Rome, Bulzoni. 53-75.

Blanche-Benveniste, C. (2012). « Postface ». Groupe de Fribourg (éd.), Grammaire de la période. Berne, Peter Lang. 337-351.

Blanche-Benveniste, C., Deulofeu J., Stéfanini J., Van den Eynde K., (1984). Pronom et syntaxe. L'approche pronominale et son application au français. Paris, SELAF.

Blanche-Benveniste, C., Bilger M., Rouget C., Van den Eynde K., Mertens P., (1990). Le français parlé : études grammaticales. Paris, CNRS.

Blanche-Benveniste, C., Willems, D. (2007). « Un nouveau regard sur les verbes faibles ». Bulletin de la Société Linguistique de Paris 102-1. 217-254.

Bolinger, D. (1984). « Intonational Signals of Subordination ». Berkeley Linguistics Society 10. 401-414.

Bril, I., Rebuschi, G., (éds). (2006). Coordination et subordination : typologie et modélisation. Faits de langues 28.

Corminboeuf, G.. (2007). « Coordination, subordination, corrélation ou énonciation autonome? Une analyse syntaxique des constructions du type Que je bouge (et) il me ramènera vite à l'ordre ». Tranel 47. 177-194.

Corminboeuf, G. (2009). L'expression de l'hypothèse en français. Entre hypotaxe et parataxe. Bruxelles, De Boeck-Duculot (coll. « Champs linguistiques »).

Corminboeuf, G. (2010). « Les structures nominales à interprétation hypothétique. Format syntaxique et constantes sémantiques ». In Béguelin M.-J., Avanzi M. \& Corminboeuf G. (éds), La Parataxe, tome 2. Berne, Peter Lang. 29-46.

Corminboeuf, G. (2013). « Corrélation et rection ». In Inkova O. \& Hadermann P. (éds), La corrélation : aspects syntaxiques et sémantiques. Genève, Droz. 41-55.

Culicover, P., \& Jackendoff, R. (2005). Simpler syntax. Oxford-New-York, Oxford University Press. Debaisieux, J.-M. (2007). « La distinction entre dépendance grammaticale et dépendance macrosyntaxique comme moyen de résoudre les paradoxes de la subordination ». Faits de langue 28. 119-132. 
Debaisieux, J.-M. (2008). Linguistique descriptive et didactique des langues étrangères. D'une cohabitation heureuse à une collaboration effective. HDR, Université de Paris-10 Nanterre.

Debaisieux, J.-M. (dir.). (2013). Analyses linguistiques sur corpus : subordination et insubordination en français. Traité IC2, série Cognition et traitement de l'information, Hermès-Lavoisier.

Defrancq, B. (2005). L'interrogative enchâssée : structure et interprétation. Bruxelles, De BoeckDuculot.

Deulofeu, H.-J. (1986). « Syntaxe de que en français parlé et le problème de la subordination ». Recherches sur le français parlé 8. 79-104.

Deulofeu, H.-J. (1991). « La notion de dépendance syntaxique dans l'approche pronominale ». L'information grammaticale, n. 50. pp. 19-23.

Deulofeu, H.-J. (1999). Recherches sur les formes de la prédication dans les énoncés assertifs en français contemporain (le cas des énoncés introduits par le morphème que). Thèse d'État, Université de Paris III.

Deulofeu, H.-J. (2007). « Les consécutives construites avec tellement ont-elles une syntaxe scalaire? ». Travaux de linguistique 54. 133-146.

Foley W. A., Van Valin R. D. (1984). Functional syntax and universal grammar. Cambridge, CUP. Gachet, F. (2012). Incises de discours rapporté et autres verbes parenthétiques : une étude grammaticale. Thèse de doctorat. Université de Fribourg.

Gautier A., Pino Serrano L., Van Raemdonck D. (éds). (2010). Les limites de la rection verbale. Numéro thématique de Travaux de linguistique 60-1.

Green, G. M. (1976). Main clause phenomena in subordinate clauses. Language 52-2. 382-397.

Groupe de Fribourg. (2012). Grammaire de la période. Berne, Peter Lang.

Hadermann, P., Pierrard, M., van Raemdonck, D., Wielemans, V. (2010). « Les structures corrélatives : pour une inscription dans les sous-systèmes parataxe/hypotaxe et coordination/ subordination ». In Béguelin M.-J., Avanzi, M. \& Corminboeuf, G. (éds), La parataxe, tome 2. Berne, Peter Lang. 219-239.

Haiman J., Thompson S. (1984). « Subordination in Universal Grammar », Subordination. Proceedings of the Tenth Annual Meeting of the Berkeley Linguistic Society. Berkeley. 510-523.

Havu E., Pierrard M. (2008). « Réduction et intégration de prédications : paramètres pour l'analyse des co-prédications adjectivantes ». Discours 2, en ligne : http:// discours.revues.org/1042.

Hjelmslev, L. (1968). Prolégomènes à une théorie du langage. Paris, Minuit.

Hjelmslev, L. (1971). « La notion de rection (1939) », Essais linguistiques. Paris, Minuit. 148-160.

Kallen-Tatarova A. (2012). Etude macro-syntaxique des marqueurs discursifs : l'exemple de donc vs alors, thèse de doctorat. Université de Fribourg.

Koch, P. (1995). « Subordination, intégration syntaxique et 'oralité' ». Etudes romanes 34. 13-42.

La Fauci, N. (1999). «Fonctions syntaxiques », communication présentée au $23^{\text {ème }}$ Colloque International de Linguistique Fonctionnelle (Lugano, 1999), inédit (en ligne).

Lazard, G. (2011). Horizons de la linguistique. Bulletin de la Société de linguistique de Paris, t. CVI, fasc. 1. 39-94.

Lehmann, C. (1988). « Towards a typology of clause linkage ». In J. Haiman \& S. A. Thompson (éds), Clause combining in grammar and discourse. Amsterdam / New York, Benjamins. 181-225. 
Kabatek, J., Obrist, P., Vincis V. (2010). «Clause-linkage techniques as a symptom of discourse traditions : methodological issues and evidence from Romance languages ». In Dorgeloh H. \& Wanner A. (eds), Syntactic Variation and Genre. Berlin-New York, Mouton de Gruyter. 247-275.

Milewski T. (1967). « La structure de la phrase dans les langues indigènes de l'Amérique du nord ». Etudes typologiques sur les langues indigènes de l'Amérique. Krakòv, Polska Academia Nauk. 70-106.

Nichols J. (1986). « Head-marking and dependent-marking grammar ». Language 62. 56-119.

Raible, W., (1992). Junktion. Eine Dimension zwischen Aggregation und Integration. Winter, Heidelberg. Raible, W. (2001). « Linking clauses ». Haspelmath, M., König E., Oesterreicher W. \& Raible W. (dir.), Language Typology and Language Universals / La typologie des langues et les universaux linguistiques / Sprachtypologie und sprachliche Universalien, vol. 20-1. Berlin / New York, de Gruyter. 590-617.

Rebuschi, G. (2001). « Coordination et subordination. Première partie : la co-jonction restreinte ». Bulletin de la société de linguistique de Paris, XCVI/1. 23-60.

Rebuschi, G. (2002). « Coordination et subordination. Deuxième partie : vers la co-jonction généralisée ». Bulletin de la société de linguistique de Paris XCVII/1. 37-94.

Sabio F. (2011). Syntaxe et organisation des énoncés - Observations sur la grammaire du français parlé. Mémoire d'Habilitation à diriger des Recherches. Université de Provence.

Seiler, H. (1988a). The Dimension of participation. Guadalajara / México, Universidad de Guadalajara.

Seiler, H. (1988b). Die universalen Dimensionen der Sprache : eine vorläufige Bilanz. Köln.

Smessaert, H., Cornillie B., Divjak D., Van den Eynde K. (2005). « Degrees of clause integration. From endotactic to exotactic subordination in Dutch ». Linguistics 43-3. 471-529.

Van Raemdonck D. (éds). (2008). Modèles syntaxiques. La syntaxe à l'aube du XXIe siècle. Berne, Peter Lang.

Verstraete, J.-C. (2007). Rethinking the coordinate-subordinate dichotomy : interpersonal grammar and the analysis of adverbial clauses in English. Berlin, Mouton de Gruyter.

Wiesmath, R. (2006). Le français acadien. Analyse syntaxique d'un corpus oral recueilli au NouveauBrunswick/Canada. Paris, L'Harmattan.

\section{NOTES}

1. Raible (2001: 596) : "Since all languages have clauses, and since all languages have to fulfill the task of linking individual propositions into larger wholes, the two extreme solutions cannot but exist in all languages: at one end of the scale, at the pole of aggregation, there are two clauses without explicit linking. What remains at the other end of the scale, the pole of integration, is one totally integrated single clause to which, in turn, another one may be added, thus giving the scale the shape of a Moebius strip ".

2. Les exemples de Wiesmath reproduits sont des exemples fabriqués que l'auteur utilise pour exposer le système de Raible, mais dans son ouvrage elle étudie la jonction dans un corpus oral de français acadien. Les exemples de Koch sont tirés d'une conversation spontanée en famille ( dîner ») et d'un cours magistral (« cours »), deux genres d'oral volontairement très différents. Nous reproduisons en note quelques extraits de Raible (1992, 2001), en anglais et en allemand qui nous paraissent instructifs quant à la démarche de l'auteur. 
3. Raible (1992: 20) : «Mit dem Übergang vom verbalen in den nominalen Bereich wird die Integration in den übergeordneten Satz nun noch um einen Grad stärker - und spätestens hier dürfte auch klar geworden sein, warum hier als Gegenpol zur Aggregation der Begriff ,Integration' und nicht die geläufigeren Begriffe der ,Subordination' oder der ,Hypotaxe' verwendet werden $»$.

4. Raible (1992 : 28) : «Während auf den Ebenen I-V (...) jeweils verbale, satzwertige Einheiten zu anderen satzwertigen Einheiten in Beziehung gebracht werden, werden auf den Ebenen VI und VII die betreffenden Relationen in Form von Nominalgruppen integriert ».

5. Raible (1992 : 25) : « Die beiden komplementären Prinzipien, die eine Dimension konstituieren, bilden dadurch ein Kontinuum, dass sich in dem Masse, in dem da seine der beiden schwächer wird, das andere sich verstärk - freilich so, dass an jeder Stelle des Kontinuums, wenn auch in je unterschiedlichem Ausmass, beide Prinzipien wirksam bleiben. Dort, wo beide Prinzipien gleich stark sind, liegt der Wendepunkt des Kontinuums bzw. der Dimension ».

6. Raible (2001: 596): «(..) instead of prepositional noun groups one could use simple prepositions like in : Wegen ihrer Krankheit blieb Joan heute zu Hause ».

7. Raible (1992: 29) : «Am einen Extrem, dem Pol der Aggregation, stehen zwei Sätze unverbunden nebeneinander. Am anderen Extrem bleibt ein einziger, völlig integrierter Satz übrig ». Mais Raible, Koch et Wiesmath ne proposent pas d'exemplification de cette technique ; on peut supposer que il ne veut pas partir constitue une phrase intégrée.

8. Les modèles macro-syntaxiques que nous exposerons au $\S 2$. ont notamment pour objectif de distinguer - au moyen de tests et de critères comme celui d'autonomie - des constructions rectionnelles vs non-rectionnelles, c'est-à-dire de déterminer s'il y a (ou non) une relation grammaticale sise entre deux unités.

9. Pourtant, selon Lazard (2011: 41) : «il n'y a pas de catégories interlangues. »

10. Culicover \& Jackendoff prennent leurs distances avec la grammaire générative. Mais il reste un substrat théorique commun. A ce propos, La Fauci (1999) montre que la grammaire générative n'a jamais vraiment cessé de réduire la syntaxe "aux deux critères de l'ordre sériel et des constituants ", ceci en dépit de la remise à jour constante de la théorie. Ce conservatisme de fait est peut-être un obstacle à une théorie des dépendances syntaxiques.

11. Parler de « rection» ( $\$ 3$, infra), c'est en revanche régler au coup par coup de multiples problèmes de segmentation du flux discursif, entreprise qui requiert une définition opératoire du concept.

12. Koch réserve une catégorie pour la nominalisation (le $\mathrm{N}$ désignation ci-dessous) qui serait la modalité de jonction la plus intégrative (mis à part le cas de la " phrase intégrée ») :

vous notez que /la désignation de Ganelon / est imposée par les barons. [oral, cours, cité par Koch]

13. Raible (1992: 25) : «Die Techniken gehen ineinander über ».

14. Koch utilise la notation $\mathrm{IV}^{*}$ pour situer l'infinitif ("subordination à verbe non conjugué ») entre les niveaux IV et V, mais plus proche de IV, tout en conservant la gradation de Raible (1992). L'auteur donne cet exemple :

l'Allemagne de l'Est paye les étudiants français / pour aller chez elle. [oral, dîner, cité par Koch] Selon Koch, l'intégration est plus forte que dans IV, parce que d'une part le syntagme nominal les étudiants français est objet du premier verbe et sujet de l'infinitif, et d'autre part la forme verbale non fléchie (aller) s'appuie sur la forme fléchie paye.

15. Nous avons fait le choix de laisser de côté d'autres approches voisines, comme les travaux de l'équipe de Florence autour d'E. Cresti ou de Paris III autour de M.-A. Morel.

16. Mais il est important de noter que dans les premières publications portant sur la " complémentation verbale ", Blanche-Benveniste (1981:61) parle de «trois degrés de relation entre un complément et un verbe » : un degré fort (valence), un degré faible (rection) et un degré nul (associé). Dans Blanche-Benveniste \& al. (1984 : 37), les auteurs affirment que « il peut y avoir 
des degrés d'intégration au verbe plus ou moins fort ». Ce n'est donc qu'à partir de BlancheBenveniste \& al. (1990) que la différence entre valence, rection et association n'est plus vue comme graduelle, mais différentielle. Il semblerait donc que ce soit la découverte de la dimension macro-syntaxique qui soit à l'origine du passage de la conception graduelle à la conception discrète.

17. Les modèles macro-syntaxiques ne sont pas cantonnés à une seule langue : on peut penser au laboratoire Lablita à Florence qui travaille sur l'italien. Et il suffit de se souvenir que l'Approche pronominale, à la base du module micro-syntaxique de la variante aixoise, trouve son origine dans la description des langues africaines (les travaux de K. Van den Eynde). Cependant, les approches macro-syntaxiques n'ont pas d'ambition typologique déclarée.

18. L'expression est de Michel Serres, à propos de la distinction que tentent de faire les paléoanthropologues (en manque de critères déterministes) entre Hommes et grands singes.

19. Smessaert \& al. (2005: 474) soulignent de même que « Maximal integration (embedding) is standardly associated with the shift from finite to nonfinite verb forms »; ils précisent également que pour Hopper \& Traugott: «infinitives and participles are the endpoint of an evolution towards maximal integration into the main clause». Smessaert \& al. accordent pourtant très peu de crédit à ce critère, qu'ils ne retiennent d'ailleurs pas pour leur étude.

20. La dichotomie syntaxe interne/externe est utilisée par les chercheurs du groupe aixois. La syntaxe interne porte sur les unités linguistiques se trouvant à l'intérieur d'un syntagme: relations syntaxiques entre ces unités et catégorie de ces unités. Elle s'oppose à la syntaxe externe, qui décrit la relation syntaxique entre un syntagme et l'unité à laquelle il est relié. La syntaxe externe de pour la calmer dans l'énoncé j'ai tout essayé pour la calmer se résume au fait d'être régi par le verbe essayer. Au niveau de la syntaxe interne, pour construit le syntagme verbal la calmer et la est complément du verbe calmer.

21. Les rapports $[\mathrm{V}<=\mathrm{A}]$ représentent les rapports entre un Verbe et son Argument.

22. L'importance du critère intonatif dans l'étude des relations entre constructions n'est plus à démontrer (Bolinger 1984, Haiman \& Thompson 1984, Avanzi 2012), bien qu'il reste peut-être à mieux mettre en correspondance le plan supra-segmental et le plan segmental. Nous laissons cet aspect de côté pour étudier le rapport entre syntaxe et sémantique, d'une part, entre marqueur morphologique et relation syntaxique, d'autre part, et entre syntaxe interne et syntaxe externe, pour finir.

23. Il est important de préciser que la distinction entre valence et « simple rection » ne recouvre pas une différence de degré de dépendance: un élément valenciel n'est pas plus régi qu'un élément de simple rection. Cela signifie que la prise en compte de la dimension lexicale n'est pas interprétée comme conduisant à deux niveaux différents de dépendance.

24. Cf. Rebuschi 2001-2002, Benzitoun \& Sabio 2010, Béguelin, Avanzi \& Corminboeuf (2010), Berrendonner 2012, etc. Mais aussi le numéro 28 de la revue Faits de langues (2006) intitulé "Coordination et subordination: typologie et modélisation", le numéro 60 de Travaux de linguistique (2010) intitulé « Les limites de la rection verbale».

\section{RÉSUMÉS}

On peut distinguer deux approches sur les dépendances syntaxiques : l'une, majoritaire, postule un continuum allant de l'intégration à la juxtaposition. L'autre adopte en revanche une vision non graduelle de l'intégration syntaxique. Dans un premier temps, nous présentons les modèles 
qui postulent un continuum d'intégration syntaxique. Dans un second temps, l'étude présente des modèles qui postulent l'existence de deux modes d'organisation des énoncés, un niveau micro-syntaxique et un niveau macro-syntaxique: ces approches, fondées notamment sur le concept de « rection », adoptent une perspective non graduelle de l'intégration syntaxique, c'està-dire qu'elles ne distinguent pas de degrés de dépendance grammaticale. Notre étude confronte les approches gradualistes et les approches non gradualistes, avec pour finalité de dégager les implications théoriques pour une modélisation des dépendances syntaxiques.

There are two ways of analysing syntactic dependencies: the most common one assumes a continuum from integration to juxtaposition. The second one adopts a non-gradual perspective of syntactic integration. At first, we present models which postulate a continuum of syntactic integration. In a second step, we present two frameworks that postulate the existence of two modes of utterances' organization, a micro-syntactic level and a macro-syntactic level: these approaches, based in particular on the concept of government ("rection" in French), adopt a nongradual perspective of syntactic integration, i.e. they do not distinguish degrees of grammatical dependency. Our study compares the gradual and non-gradual approaches, with the aim of identifying the theoretical implications for modeling syntactic dependencies.

INDEX

Mots-clés : dépendance, subordination, rection, macro-syntaxe, contrainte

Keywords : dependency, subordination, government, macro-syntax, constraint

\section{AUTEURS}

\section{GILLES CORMINBOEUF}

Fonds national suisse de la recherche scientifique (FNRS)

\section{CHRISTOPHE BENZITOUN}

Université de Lorraine \& Laboratoire ATILF (CNRS UMR 7118) 